\title{
Aproximación jurídica a la regulación cooperativa de los instrumentos financieros no tradicionales y su utilización en el sector agroalimentario español $^{1}$
}

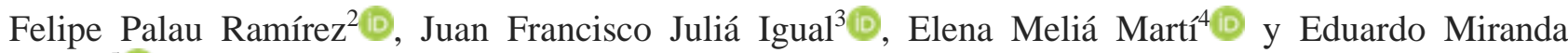 \\ Ribera $^{5}(0)$
}

Recibido: 5 de mayo de 2021 / Aceptado: 9 de mayo de 2021 / Publicado: 9 de julio de 2021

Resumen. El presente trabajo tiene como objetivo analizar si las sociedades cooperativas españolas del sector agroalimentario utilizan las obligaciones, participaciones especiales y títulos participativos como método alternativo de financiación. Para ello, se analizaron los aspectos jurídicos más relevantes y la incidencia contable de estos instrumentos financieros en las sociedades cooperativas. Posteriormente, se analizaron las cuentas anuales de las 10 mayores sociedades cooperativas agroalimentarias por volumen de facturación y se compararon estos resultados con los obtenidos por las 10 mayores sociedades de responsabilidad limitada y anónimas. Lamentablemente, a excepción de dos casos concretos, ninguna de las sociedades analizadas ha hecho uso de este tipo de instrumentos financieros, cuando sí están necesitadas de financiación ajena, dado su elevado endeudamiento, por lo que, cabría plantearse sí, quizás, sería necesario adaptar la legislación estatal y autonómica en materia de cooperativas para regular nuevas formas de financiación que fueran más atractivas para las sociedades cooperativas del sector agroalimentario.

Palabras clave: Cooperativas; Obligaciones; Participaciones especiales y títulos participativos.

Claves Econlit: K10; M40; 013; 016.

\section{[en] Legal approach to the cooperative regulation of non-traditional financial instruments} and their use in the Spanish agri-food sector

\begin{abstract}
The aim of this paper is to analyze if the Spanish agri-food cooperatives make use of obligations, special participations and participatory titles as an alternative financial system. Therefore, the main legal and accounting aspects of these financial instruments are going to be considered in order to identify their most relevant characteristics. In order to conduct this research, it was necessary to analyze the annual accounts of some cooperative companies of the agri-food sector. Additionally, with the aim of being more accurate in our analysis, we compared the obtained results with some S.R.L and S.A. from the agri-food sector. However, none of the selected societies had made used of obligations, special participations and participatory titles, so we think that it might be necessary to adapt our cooperatives legislation in order to develop new financial instruments which were better for the cooperatives of the agrifood sector.
\end{abstract}

Keywords: Cooperatives; Obligations; Special participations and participatory titles.

Sumario. 1. Introducción. 2. Objetivos y metodología. 3. Régimen jurídico. 4. Resultados obtenidos. 5. Conclusiones. 6. Referencias bibliográficas.

Cómo citar. Palau Ramírez, F.; Juliá Igual, J.F.; Meliá Martí, E.; Miranda Ribera, E. (2021) Aproximación jurídica a la regulación cooperativa de los instrumentos financieros no tradicionales y su utilización en el sector agroalimentario español. REVESCO. Revista de Estudios Cooperativos, vol. 139, e76633. https://dx.doi.org/10.5209/reve.76633.

1 El presente trabajo se enmarca dentro del Proyecto de I+D de la Universitat Politècnica de València denominado "Innovaciones en la gestión empresarial de las cooperativas agroalimentarias", financiado por CAJAMAR.

2 Universitat Politècnica de València, España.

Dirección de correo electrónico: fpalau@upv.es.

Universitat Politècnica de València, España.

Dirección de correo electrónico: ifjulia@esp.upv.es

4 Universitat Politècnica de València, España.

Dirección de correo electrónico: emeliam@esp.upv.es.

5 Universitat Politècnica de València, España.

Dirección de correo electrónico: edmiri@ doctor.upv.es. 


\section{Introducción}

Las empresas cooperativas del sector agroalimentario europeo, con el devenir de los años, han alcanzado un importante desarrollo tanto estructural como financiero. De hecho, el modelo cooperativo ha permitido a los agricultores un mejor acceso a los mercados, tanto a nivel abastecimiento de inputs como de venta de sus productos, lo que les ha permitido mejorar tanto en términos de seguridad como de precios (Bermard et.al. 2008; Ito et al, 2012), permitiéndoles continuar con la explotación de sus tierras y su actividad de forma rentable (Cook, 1995). No debe olvidarse que las primeras cooperativas surgieron como "colectivos de personas unidas por una necesidad común, unidas (para) hacer frente conjuntamente a esa necesidad" (Fajardo, 2011). Esta definición fue ampliada, posteriormente, por la Alianza Cooperativa Internacional (en adelante ACI), que configuró a las cooperativas como una "sociedad con capital variable y estructura y gestión democráticas que asocia a personas, en régimen de libre adhesión y baja voluntaria, para realizar actividades económicas y sociales de interés común y de naturaleza empresarial, reputándose los resultados económicos a los socios una vez atendidos los fondos comunitarios, en función de la actividad cooperativizada que realizan" (Macías, 2016). Esta definición es similar a la configurada por el legislador español que las define como "una sociedad constituida por personas que se asocian, en régimen de libre adhesión y baja voluntaria, para la realización de actividades empresariales, encaminadas a satisfacer sus necesidades y aspiraciones económicas y sociales, con estructura y funcionamiento democrático conforme a los principios formulados por las alianza cooperativa internacional" (art. 1 Ley 27/1999, de 16 de julio, de Cooperativas).

Sin embargo, el modelo cooperativo presenta algunas limitaciones, entre las que por su enorme repercusión en la viabilidad del modelo destacan las de índole financiero. De hecho, el propio contexto en el que operan estas empresas las obliga a la realización de continuas inversiones en economías de escala y de alcance, con las que las empresas persiguen ganar eficiencia en aras de dar unos mejores resultados a sus socios y mantener su competitividad en un sector muy exigente como el agroalimentario, tales como inversiones en industrias con las que captar valor añadido, desarrollo de marcas, entrada en mercados internacionales, etc. (Chaddad \& Cook, 2004; Cook and Iliopoulos, 2000; Grashuis, 2018). Así, estas empresas requieren de importantes flujos financieros de inversión, los cuales en muchos casos no han sido fácilmente alcanzables para las cooperativas tradicionales, que se han caracterizado en muchos casos por tener un elevado porcentaje de socios que rehúyen incrementar su inversión, aspecto ampliamente analizado por la academia, y que está íntimamente relacionado con algunos de sus problemas inherentes: problema del polizón, del horizonte, portfolio, o de monitorización (Harte, 1997; Cook 1995; Ortman, 2007; Nilsson et al, 2012).

Así, el llamado problema del horizonte aflora cuando los socios encuentran pocos incentivos a la inversión en las cooperativas, debido principalmente, a que los beneficios esperables se generan en un horizonte temporal en el que al menos en parte, los socios no van a estar presentes. El problema del polizón o free-rider, se hace visible con la incorporación de nuevos socios, ya que éstos tendrán los mismos derechos a la hora de disfrutar los servicios de la cooperativa, o ante la distribución de resultados, a pesar de no haber tenido que realizar inversiones equivalentes a las de los socios preexistentes (los cuales además de capital, han ido contribuyendo gracias a la dotación de reservas a la creación de un patrimonio colectivo considerable), lo que hace que la inversión en la cooperativa pierda interés, excepto en el caso en que para la entrada en la cooperativa el nuevo socio deba satisfacer una cuota de entrada, la cual se destina íntegramente al fondo de reserva obligatorio. Por último, el problema del portfolio se refiere a la preferencia de los socios por mantener bajos niveles de inversión en la cooperativa por la falta de alineación entre el nivel de riesgo del socio, y en consecuencia de la cartera de inversiones que él preferiría (portfolio), y el de la cooperativa.

A los problemas expuestos hay que añadir que una buena parte de los excedentes de las cooperativas se quedan en las mismas en forma de reservas, no repercutiendo en el socio en caso de baja, a lo que se une la falta de actualización del valor de su participación en el capital, que hace que cuando el socio causa baja, la cantidad reembolsada no refleje en ningún caso el incremento de valor que haya podido experimentar la sociedad en el periodo en el que éste estuvo en la cooperativa (Cook e Iliopoulos, 2013).

Todo ello ha derivado en que muchas cooperativas tradicionales sufran limitaciones en la disponibilidad de capital, lo que ha conducido al desarrollo de innovaciones estructurales de distinta índole, con objeto de hacer más atractiva la inversión en la cooperativa, y de esta forma salvar ese problema. Así, han aparecido modelos diversos a nivel europeo, como las cooperativas de inversión proporcional (en las que los socios contribuyen al capital en función de la actividad), las cooperativas con socios-inversores (que solo admiten como socios a los productores, pero en las que se distribuyen resultados en base al capital), las cooperativas con inversores (que admiten tanto a socios productores como a inversores, existiendo dos clases de participaciones en el capital, para los productores y para los inversores), las cooperativas de nueva generación (con derechos de entrega de producción transmisibles, lo que aporta liquidez y la posibilidad de que los derechos de entrega se puedan vender a un valor actualizado en el mercado secundario), o cooperativas que han creado una sociedad de capital para atraer a través de ella capital (Nilsson, 1999; 
Chaddad y Cook, 2004; Beckum y Bijman, 2006). Y en el caso más extremo encontramos cooperativas que han optado por la desmutualización, convirtiéndose en sociedad de capital, con su consecutiva salida a bolsa. En este escenario se ha acuñado el término "cooperativa híbrida", para referirse a aquellas cooperativas que han adoptado alguna de las características de las sociedades de capital, pero que mantienen la identidad cooperativa (Royer, 1999). De hecho, el proceso de hibridación que han sufrido muchas cooperativas europeas es considerado por algunos como la respuesta de las cooperativas a los procesos de globalización y necesidad de financiación que han caracterizado el final del siglo XX, habiendo adquirido diferentes connotaciones dependiendo del país y de su legislación (Battilani y Schröter, 2012). Igualmente, cabe reconocer que este tipo de modelos ha permitido incrementar la financiación de estas entidades propiciando su crecimiento, el desarrollo de su innovación y una mayor capacidad para abordar procesos de diversificación y de internacionalización, ahora bien, todo ello, sin perjuicio de los riesgos degenerativos de los valores y principios cooperativos.

En España el crecimiento de las cooperativas se ha dado mayoritariamente a través de fórmulas como la cooperativa de segundo grado (6 de las 10 mayores cooperativas agroalimentarias españolas adoptan esta configuración), y también en menor medida de procesos de fusión (algunos han sido determinantes en la configuración de algunas de las cooperativas más importantes del panorama español, como es el caso de ALIMER, S. Coop, resultado de la fusión de 8 cooperativas y una SAT). Los modelos híbridos son aún un caso poco común en el panorama cooperativo español, a pesar de que algunas legislaciones cooperativas han incluido en su articulado fórmulas en este sentido. Así, la propia Ley 27/1999 de cooperativas, y algunas leyes regionales permiten a los inversores externos tomar una participación en el capital de la cooperativa, a través de opciones tales como las Cooperativas mixtas, recogidas en el artículo 107 de la Ley 27/1999 y en algunas leyes regionales como la del País Vasco (art. 136), Castilla-La Mancha (art. 152), Región de Murcia (art. 128), Asturias (art. 186) y Navarra (art. 79), o como en el caso de la "Persona inversora", reflejado en la legislación regional andaluza (art. 25). En España contamos con ejemplos de modelos organizacionales que pueden considerarse híbridos, al utilizar una sociedad de capital como sociedad instrumental central para el desarrollo de su actividad principal y complementaria, incorporando la misma socios exclusivamente de capital (inversores externos). Este es el caso de Indulleida S.A., y Corporación Alimentaria de Guissona S.A, las cuales pese a asumir la sociedad central la fórmula de sociedad anónima, siguen considerándose de base cooperativa, al mantenerse el control de la sociedad de capital en manos de las cooperativas de origen y/o sus socios, preservándose por tanto su identidad cooperativa (Meliá-Martí et. al, 2018).

\section{Objetivos y metodología}

El presente trabajo tiene como objetivo analizar si las cooperativas agroalimentarias españolas, utilizan las obligaciones, las participaciones especiales y los títulos participativos como sistema alternativo de captación de recursos financieros. Para ello, en primer lugar, se analizará su régimen jurídico y sus características, con el objetivo de conocer las implicaciones jurídicas derivadas de la facultad de las sociedades cooperativas para emitir estos títulos como método alternativo de financiación. Igualmente importante es analizar e identificar las implicaciones contables que la emisión de estos títulos tienen en estas sociedades, máxime cuando el legislador, mediante la Orden EHA/3360/2010, de 21 de diciembre, por la que se aprueban las normas sobre los aspectos contables de las sociedades cooperativas (Publicada en "BOE" núm. 316, de 29 de diciembre de 2010), introduce una serie de consideraciones contables que son directamente aplicables a la emisión de este tipo de instrumentos financieros. De esta forma, el análisis de la mencionada Orden EHA/3360/2010 servirá de ayuda para una mejor detección de estos instrumentos financieros en las cuentas anuales de las cooperativas.

Una vez analizados los aspectos jurídicos y contables más relevantes de la emisión de este tipo de instrumentos financieros por parte de las sociedades cooperativas, se estudia la implantación de los mismos a través del análisis de las cuentas anuales de las sociedades cooperativas agroalimentarias con mayor volumen de facturación (top 10). Por su relevancia, se han incluido en el análisis Sociedades Agrarias de Transformación (SAT). La reducción de la muestra objeto de análisis al top 10 se debe a que este tipo de instrumentos son comunes entre las empresas de gran dimensión, siendo muy usuales entre las empresas del IBEX, siendo prácticamente inexistentes en las pymes, que recordemos constituyen la mayor parte de entidades asociativas agroalimentarias españolas.

A su vez, se ha estudiado la presencia de estos instrumentos en sus sociedades homólogas, basadas en el capital (anónimas y limitadas), igualmente en el top 10 de cada una de ellas, con objeto de contrastar si pueden existir diferencias en su emisión derivadas de una diferente gestión consecuencia de su forma jurídica.

Aunque el legislador faculta a las sociedades de capital para emitir cualquier tipo de título en aras de captar financiación, en este trabajo la búsqueda se ha centrado exclusivamente en el análisis de las obligaciones, participaciones especiales y los títulos participativos, en consonancia con la realizada en las 
cooperativas. Se da la circunstancia de que las participaciones especiales y los títulos participativos son característicos exclusivamente de las sociedades cooperativas, aunque, ello, no es óbice para afirmar que las sociedades de capital o las SAT no puedan emitir instrumentos con similares características. Así, persiguiendo analizar la existencia de emisiones de títulos análogos a las participaciones especiales y títulos participativos por parte de las sociedades de capital, y dado que contablemente se integran en la misma partida en la que se refleja la emisión de obligaciones, se ha analizado esta cuenta en las sociedades de capital y en las SAT.

Para proceder a la recopilación de datos de las cuentas anuales se plantea una metodología de trabajo que se ha dividido en dos fases. En una primera fase, se ha realizado una recopilación y selección de las empresas con actividad en el sector agroalimentario, al objeto de poder seleccionar el top 10 por facturación tanto de las sociedades cooperativas como de las de capital. Los criterios de búsqueda en la Base de Datos SABI han sido los siguientes: (1) Forma jurídica, (2) Sector de actividad y (3) Volumen de facturación. De forma más concreta, la forma jurídica seleccionada ha sido la de entidad asociativa (cooperativa o SAT), sociedad anónima y sociedad de responsabilidad limitada; al objeto de establecer los sectores de actividad concreto en los que operan las cooperativas agroalimentarias españolas, se efectuó una consulta a Cooperativas Agroalimentarias, entidad representativa de las cooperativas agroalimentarias españolas, la cual nos facilitó el siguiente listado de códigos CNAE, que son los que se han utilizado: (01) Agricultura, ganadería, caza y servicios relacionados con las mismas, (02) Silvicultura y explotación forestal, (03) Pesca y acuicultura, (10) Industria de la alimentación, (11) Fabricación de bebidas, (4617) Intermediarios del comercio de productos alimenticios, bebidas y tabaco, (462) Comercio al por mayor de materias primas agrarias y de animales vivos, (463) Comercio al por mayor de productos alimenticios, bebidas y tabaco, (4776) Comercio al por menor de flores, plantas, semillas, fertilizantes, animales de compañía y alimentos para los mismos en establecimientos especializados, (5210) Depósito y almacenamiento, (6622) Actividades de agentes y corredores de seguros, (6920) Actividades de contabilidad, teneduría de libros, auditoría y asesoría fiscal, (7022) Otras actividades de consultoría de gestión empresarial, (8211) Servicios administrativos combinados, (9499) Otras actividades asociativas. Por último, el volumen de facturación mínimo fijado ha sido de 10.000.000 de euros.

En una segunda fase, se han segmentado por forma jurídica, y se ha extraído información de sus balances al respecto del uso de los instrumentos financieros objeto de estudio. (en adelante Fase 2). A tal fin, se han extraído datos que nos permiten conocer de forma directa los siguientes datos de carácter contable y financiero: 1. obligaciones y otros valores negociables a largo plazo; 2. Obligaciones y otros valores negociables a corto plazo; 3. Deudas con características especiales a largo plazo El principal motivo por el que se ha filtrado por estos campos radica en que la Base de Datos seleccionada únicamente permite filtrar por esos campos y, según la Orden EHA/3360/2010, la hipotética contabilización de las obligaciones, participaciones especiales y títulos participativos debería estar registradas en esas cuentas.

La Base de Datos SABI utilizada en el presente trabajo contiene información jurídica y económicofinanciera de más de 2 millones de entidades españolas, siendo muy utilizada en investigación por incluir a un porcentaje muy elevado y representativo de las empresas que operan en el territorio español (78\% en sociedades anónimas, $65 \%$ de las sociedades de responsabilidad limitada y $31 \%$ de las cooperativas) (tabla $1)$.

Tabla. 1. Alcance de la base de datos SABI

\begin{tabular}{|c|c|c|c|c|}
\hline \multirow[b]{2}{*}{$\begin{array}{l}\text { Tipo de } \\
\text { sociedad }\end{array}$} & \multirow{2}{*}{$\begin{array}{c}\text { Número de } \\
\text { Empresas activas } \\
\text { según el DIRCE } \\
\text { (2018) }\end{array}$} & \multicolumn{2}{|c|}{ Número de empresas incluidas en SABI } & \multirow[b]{2}{*}{$\begin{array}{c}\text { \% empresas activas } \\
\text { en SABI/empresas } \\
\text { activas según } \\
\text { DIRCE }\end{array}$} \\
\hline & & Totales & Activas & \\
\hline SA & 78.866 & 127.653 & 61.336 & $78 \%$ \\
\hline SL & 1.181 .391 & 1.508 .100 & 771.427 & $65 \%$ \\
\hline SCoop & 20.707 & 8.027 & 6.341 & $31 \%$ \\
\hline
\end{tabular}

Fuente: Elaboración propia a partir del Directorio Central de Empresas, obtenida a partir de la base de datos del INE (INEbase), y de la base de datos SABI. 


\section{Régimen jurídico}

Siguiendo con el iter procedimental expuesto en la metodología y dado que el objeto del presente trabajo se centra en España, se procede a analizar, brevemente, el régimen jurídico aplicable, en materia de sociedades cooperativas conforme a la normativa estatal y autonómica, a la emisión de obligaciones, participaciones especiales y títulos participativos por parte de las sociedades cooperativas. Para ello, a modo introductorio, interesa poner de manifiesto que, tradicionalmente, las sociedades cooperativas se han caracterizado por financiarse mediante: 1. aportaciones sociales, entendiendo como tales la contribución de cada socio al patrimonio neto, así como el "título en el que se materializa la cuota social"(Pastor Sempere, 2011; Macías Ruano, 2016), con la salvedad de que no todas las aportaciones de los socios se incorporarán en el capital social (Pendón Meléndez, 2019); 2. autofinanciación, integrada por los excedentes generados por la cooperativa, así como los fondos de reserva obligatorios, fondo de educación y promoción y fondo de reserva voluntario (Martín Castro, 2019); 3. financiación externa tradicional, como, por ejemplo, préstamos bancarios o pólizas de crédito; y no tradicional como, por ejemplo, las obligaciones, participaciones especiales y títulos participativos (Morillas Jarillo y Feliú Rey, 2018; Peinado Gracia y Vázquez Ruano, 2019). Asimismo, para un mejor entendimiento del presente trabajo se detallan, a continuación, en la Tabla 2, todas las leyes, tanto estatales como autonómicas, en materia de cooperativas que regulan la figura de las obligaciones, participaciones especiales y títulos participativos.

Tabla. 2. Instrumentos financieros integrantes del capital social de las cooperativas en España

\begin{tabular}{|c|c|c|c|}
\hline LEY & Obligaciones & $\begin{array}{l}\text { Participacion } \\
\text { es especiales }\end{array}$ & $\begin{array}{l}\text { Títulos } \\
\text { participativos }\end{array}$ \\
\hline $\begin{array}{l}\text { Ley del Principado de Asturias 4/2010, de } 29 \text { de junio, } \\
\text { de Cooperativas, en adelante LCAst }\end{array}$ & Art. 95.2 & Art 94 & Art. 95.4 \\
\hline $\begin{array}{c}\text { Ley } 11 / 2010 \text {, de } 4 \text { de noviembre, de Cooperativas de } \\
\text { Castilla-La Mancha, en adelante LCCLM }\end{array}$ & Art. 83.3 & Art. 84 & Art. 83.5 \\
\hline $\begin{array}{l}\text { Ley 4/2002, de } 11 \text { de abril, de Cooperativas de la } \\
\text { Comunidad de Castilla y León, en adelante LCCyL }\end{array}$ & Art. 70.3 & Art.70.2 & Art. 70.1 \\
\hline $\begin{array}{l}\text { Ley 5/1998, de } 18 \text { de diciembre, de Cooperativas de } \\
\text { Galicia, en adelante LCGal }\end{array}$ & Art. 65.4 & Art.65.4 & Art. 65.5 \\
\hline $\begin{array}{c}\text { Ley 11/2019, de } 20 \text { de diciembre, de Cooperativas de } \\
\text { Euskadi, en adelante LCEus }\end{array}$ & Art. 68.4) & Art. 68.4 & Art. 68.4 \\
\hline $\begin{array}{l}\text { Ley } 12 / 2015 \text {, de } 9 \text { de julio, de cooperativas de Cataluña, } \\
\text { en adelante LCCat }\end{array}$ & Art. 77.4 & Art. 77.1 & Art. 77.2 \\
\hline $\begin{array}{c}\text { Ley 4/1999, de } 30 \text { de marzo, de Cooperativas de la } \\
\text { Comunidad de Madrid, en adelante LCMad }\end{array}$ & Art. 57.3 & Art. 57.3 & Art. 57.4 \\
\hline $\begin{array}{l}\text { Ley 14/2011, de } 23 \text { de diciembre, de Sociedades } \\
\text { Cooperativas Andaluzas, en adelante LCAnd }\end{array}$ & Art. 62.4 & Art. 63.2 & Art. 62.5 \\
\hline $\begin{array}{c}\text { Decreto Legislativo } 2 / 2015 \text {, de } 15 \text { de mayo, del Consell, } \\
\text { por el que aprueba el texto refundido de la ley de } \\
\text { cooperativas de la Comunitat Valenciana, en adelante } \\
\text { LCCVal }\end{array}$ & Art..62.3 & Art.62.3 & Art. 62.4 \\
\hline Decreto Legislativo 2/2014, de 29 de agosto, del & Art.55.4 & Art.55.3 & Art. 55.5 \\
\hline
\end{tabular}




\begin{tabular}{|c|c|c|c|}
\hline $\begin{array}{l}\text { Gobierno de Aragón, por el que se aprueba el texto } \\
\text { refundido de la Ley de Cooperativas de Aragón, en } \\
\text { adelante LCAra }\end{array}$ & & & \\
\hline $\begin{array}{l}\text { Ley Foral 14/2006, de } 11 \text { de diciembre, de Cooperativas } \\
\text { de Navarra, en adelante LCNav }\end{array}$ & Art.45.9 & Art. 47.1.b) & Art. 45.13 \\
\hline $\begin{array}{c}\text { Ley 4/2001, de } 2 \text { de julio, de Cooperativas de La Rioja, } \\
\text { en adelante LCLRio }\end{array}$ & Art. 69.4 & Art.69.3 & Art. 69.5 \\
\hline $\begin{array}{c}\text { Ley 8/2006, de } 16 \text { de noviembre, de Sociedades } \\
\text { Cooperativas, de la Región de Murcia, en adelante } \\
\text { LCRMu }\end{array}$ & Art.74.1 & Art. 73.1 & Art. 74.2 \\
\hline $\begin{array}{l}\text { Ley 9/2018, de } 30 \text { de octubre, de sociedades } \\
\text { cooperativas de Extremadura, en adelante LCEx }\end{array}$ & Art.74.4 & Art. 74.2 & Art. 74.5 \\
\hline $\begin{array}{c}\text { Ley } 1 / 2003 \text { de } 20 \text { de marzo, de cooperativas de las Illes } \\
\text { Balears, en adelante LCIBal }\end{array}$ & Art.78.1 & Art. 77 & Art. 78.2 \\
\hline $\begin{array}{c}\text { Ley 6/2013, de } 6 \text { de noviembre, de Cooperativas de } \\
\text { Cantabria, en adelante LCCan }\end{array}$ & Art.69.1 & Art. 68.1 & Art. 69.2 \\
\hline Ley $27 / 1999$, de 16 de julio, de Cooperativas, LC & Art.54.1 & Art. 53.1 & Art. 54.2 \\
\hline
\end{tabular}

Fuente: Elaboración a través del estudio de la normativa estatal y autonómica en materia de cooperativas.

Las obligaciones han sido definidas por la doctrina mercantilista clásica como "valores mobiliarios emitidos como títulos o anotaciones en cuenta necesariamente en serie impresa o numerada, iguales, acumulables, indivisibles y transferibles, en forma nominativa o al portador, que contienen o incorporan un reconocimiento de deuda de dinero (tanto si nace con la suscripción como si los títulos se entregan a quien ya era acreedor), que incluyen la promesa dirigida a cualquier legítimo tenedor de la restitución de su nominal y pago de intereses, propia de todo préstamo" (Vicent Chuliá, 2012) o, de forma más concreta como "valores emitidos en serie o en masa, mediante los cuales la sociedad emisora reconoce o crea una deuda de dinero a favor de quienes lo suscriben" (García de Enterría, 2006). De ello se extrae que, en toda emisión de obligaciones, concurrirán dos agentes: el "emisor" o entidad que realiza la emisión y el "obligacionista" que adquiere los títulos con el objetivo de obtener un rendimiento (Vicent Chuliá, 2012).

Tradicionalmente, la emisión de obligaciones como método alternativo de financiación, se ha caracterizado por ser un sistema de captación de recursos financieros típico de las sociedades capital, en particular, las sociedades anónimas. Sin embargo, esta práctica habitual no lo era tanto en las sociedades cooperativas, por lo que, el legislador con el objetivo de fomentar su utilización promulgó la Ley 5/2015, de 27 de abril, de Fomento de la Financiación donde, entre otras, estableció una serie de disposiciones aplicables a las sociedades cooperativas como, por ejemplo, la designación del Consejo Rector como órgano competente para adoptar el acuerdo de emisión de obligaciones en aras de fomentar su utilización.

Ahora bien, es de destacar que la normativa estatal y autonómica en materia de cooperativas, es parca, por cuanto únicamente se limita a reconocer la posibilidad de las cooperativas para poder emitir obligaciones como método alternativo de financiación a los sistemas tradicionales (aportaciones de socios, reservas, etc.). Igualmente, a la hora de definir el concepto y el régimen jurídico de las obligaciones, el legislador -estatal y autonómico en materia de cooperativas- aboga por establecer una entera remisión a lo dispuesto en el Real Decreto Legislativo 1/2010, de 2 de julio, por el que se aprueba el texto refundido de la Ley de Sociedades de Capital (en adelante LSC o Ley de Sociedades de Capital). Ello nos induce a afirmar que todo lo relativo al régimen de emisión y características de las obligaciones será conforme a lo establecido en el acuerdo de emisión de la sociedad cooperativa emisora que, en todo momento, deberá respetar lo dispuesto en la Ley de Sociedades de Capital y el Real Decreto Legislativo 4/2015, de 23 de octubre, por el que se aprueba el texto refundido de la Ley del Mercado de Valores, algo que a nuestro entender podría generar, en algunas cuestiones puntuales, algún conflicto con los principios y valores cooperativos; no obstante, esta cuestión no será abordada en el presente trabajo máxime cuando va más allá del objeto del mismo. 
Las participaciones especiales, por su parte, vienen definidas en el artículo 53 de la Ley 27/1999, de 16 de julio, de Cooperativas, como un instrumento capaz de "captar recursos financieros de socios o de terceros, con el carácter de subordinados y con un plazo mínimo de vencimiento de cinco años" (Pastor Sempere, 2002). La doctrina especializada en la materia entiende que la finalidad perseguida por el legislador al establecer el régimen jurídico de las participaciones especiales consistía en "sustituir el capital variable de la cooperativa por un fijo a todos los efectos, que se integraría por las participaciones especiales, le sería de aplicación el régimen propio del capital excepto los artículos relativos a retribución, actualización, transmisión y reembolso" (Fajardo, 2005).

Por ello, al objetivo de obtener la mayor financiación posible, este tipo de instrumentos financieros se destinará tanto a los socios cooperativistas como a terceros inversores. Hay autores que incluso consideran que las participaciones especiales son "aportaciones patrimoniales realizadas por los socios o terceros, que presentan una naturaleza compleja, asimilable a los préstamos subordinados, en tanto que, por una parte, presentan notas comunes con el préstamo, aunque, por otra, están comprometidas en cierta medida en el riesgo de empresa" (Gadea, 2003). Esto nos permite, por tanto, afirmar que las participaciones especiales se caracterizan fundamentalmente por: 1 . su carácter subordinado; 2. el amplio plazo de vencimiento que deberá ser, como mínimo, de 5 cinco años o, incluso, estar vinculado a "la aprobación de la liquidación de la sociedad cooperativa, (que) tendrán la consideración de capital social" (art. 53.1 Ley 27/1999, de 16 de julio, de Cooperativas); 3 . otorgar derechos de carácter político, como por ejemplo, derecho de asistencia a la Asamblea General, pero, en modo alguno, derecho de voto; 4. y llevar aparejados derechos de carácter económico, entendiendo como tal un derecho a obtener una retribución (Pastor Sempere, 2002).

De todas las características de las participaciones especiales, adquiere especial relevancia la posibilidad de configurarlas con un plazo de vencimiento vinculado a la liquidación de la sociedad cooperativa, máxime cuando ello cuestiona su naturaleza jurídica. No obstante, resulta esclarecedor, en este sentido, lo dispuesto en el artículo 47 de la legislación navarra en materia de cooperativas. Este artículo, define de manera amplía que se entiende por fondos propios variables, capital social fijo y otros recursos propios. En lo que nos interesa para el presente trabajo, se considera capital social fijo "cualquier modalidad de deuda perpetua subordinada no exigible hasta la liquidación de la cooperativa receptora y no reembolsable con anterioridad, salvo con consentimiento expreso o tácito de (los) acreedores" (art. 47 Ley de Cooperativas de Navarra).

Esta definición, por tanto, nos permite clasificar las participaciones especiales en función de su vencimiento. De manera que, conforme a lo establecido en el acuerdo de emisión, cuya competencia exclusiva radica en la Asamblea General de la sociedad cooperativa emisora (art. 21.2 e) y 53.2 Ley 27/1999, de 16 de julio, de Cooperativas), las participaciones especiales, a efectos legales, podrán ser considerados como capital social fijo o como un pasivo financiero. No obstante, esto es matizable, ya que, como se explicará más adelante, a efectos contables cabe realizar ciertas puntualizaciones, por cuanto la consideración de las participaciones especiales como fondos propios surge del cumplimiento de dos requisitos tasados por el legislador en la Orden EHA/3360/2010.

En último lugar, los títulos participativos han sido definidos como instrumentos financieros caracterizados porque su retribución deberá consistir, esencialmente, en una participación en los resultados o en la evolución de la actividad económica de la sociedad que los emita, por lo que, algunos autores afirman que este tipo de instrumentos financieros son una variante cooperativa de los préstamos participativos (Rocafort Nicolau, 2010). Otros autores, van incluso más allá y cuestionan la necesidad de introducir los títulos participativos en el ordenamiento jurídico español, por cuanto únicamente representan una mera clase de obligaciones, que se diferencian del resto en el sistema de retribución de carácter mixto; a la remuneración de tipo fijo se añade otra de carácter variable necesariamente vinculada a la evolución de la actividad de la sociedad cooperativa emisora (Gadea et al, 2009).

Esto evidencia que la retribución de los títulos participativos representa su pilar fundamental, por cuanto le diferencia del resto de instrumentos de financiación ajena de las sociedades cooperativas y, además, plasma el elemento más atractivo para los inversores. Sin embargo, a pesar de la insistencia de la doctrina especializada en la materia en vincular la retribución de los títulos participativos a la evolución de la actividad de la sociedad cooperativas, existen determinadas Comunidades Autónomas como La Rioja, Extremadura e Islas Baleares que permiten establecer una remuneración exclusivamente de carácter fijo. Esto, en nuestra opinión, podría desvirtuar la razón de ser del instrumento financiero, por cuanto, elude/elimina su carácter participativo y lo equipara a una mera obligación.

Ulteriormente, la principal razón por la que el legislador decidió introducir la figura de los títulos participativos en el ordenamiento jurídico radica en su voluntad de "permitir que el préstamo participativo pueda incorporarse a un valor mobiliario para, esencialmente, facilitar su transmisibilidad" (Pastor Sempere, 2002). En este caso, el instrumento financiero viene caracterizado, además de por su sistema de retribución, por su condición de valor mobiliario. Al igual que las obligaciones, el legislador estatal y autonómico en materia de cooperativas no se pronuncian sobre su régimen de emisión, estableciendo que se deberá atender a lo dispuesto en el acuerdo de emisión de los títulos -cuya competencia exclusiva radica en la Asamblea General de la cooperativa (art. 21.2 e) y 54.2 Ley 27/1999, de 16 de julio, de Cooperativas)-, que, en todo 
caso deberá respetar lo dispuesto en la Ley de Sociedades de Capital y la normativa financiera vigente en materia de emisión de valores. Una vez definidos, brevemente, los diferentes instrumentos financieros susceptibles de análisis en el presente trabajo, para una mejor comprensión del trabajo, resulta menester identificar en qué cuentas contables se registrarán dentro del balance de las sociedades cooperativas las obligaciones, las participaciones especiales y los títulos participativos. Para ello es obligatoria la remisión a lo establecido en la, ya mencionada, Orden EHA/3360/2010. En esta Orden, entre otras, se establecen diferentes elementos de carácter eminentemente contable que resultan de aplicación directa para las sociedades cooperativas, en particular, la contabilización de los instrumentos financieros anteriormente definidos y, más concretamente, las participaciones especiales.

La opción del legislador por una norma ajena a la metodología y requisitos de contabilización de las participaciones especiales se debe, principalmente, a que tanto las obligaciones como los títulos participativos, en su condición de títulos de deuda se contabilizarán en una cuenta de pasivo. De manera que simplemente debe diferenciarse si estamos ante títulos con vencimiento en el corto plazo o en el largo plazo. En su caso, cuando el plazo de vencimiento sea superior a un año se utilizará la cuenta 177. Obligaciones y bonos, para las obligaciones y la cuenta 179. Deudas representadas en otros valores negociables, para los títulos participativos: ambas cuentas se integrarán dentro del pasivo no corriente de la sociedad cooperativa emisora. Cuando el plazo de vencimiento de las obligaciones y los títulos participativos sea inferior a un año se utilizará la cuenta 500. Obligaciones y bonos a corto plazo, para las obligaciones y la cuenta 505. Deudas representadas en otros valores negociables a corto plazo, para los títulos participativos, y ambas se anotarán en el pasivo corriente de la sociedad cooperativa emisora.

Esta aparente sencillez de identificación y registro contable no es tal cuando se emiten participaciones especiales. Conforme a la Norma Quinta de la, ya mencionada, Orden EHA/3360/2010, a efectos contables, la consideración de las participaciones especiales como fondos propios viene configurada por que: "1. únicamente exista obligación de reembolso en caso de liquidación de la cooperativa; y 2. no lleven aparejado el pago de una remuneración obligatoria por parte de la cooperativa". Esta configuración -que no trasciende más allá del ámbito contable-, nos permite diferenciar si se contabiliza el instrumento financiero como un pasivo financiero o como un fondo propio, en función de sus características.

Cuando se cumplan los anteriores requisitos y las participaciones especiales sean consideradas como fondos propios, la Orden EHA/3360/2010 establece que se contabilizarán en las siguientes cuentas: "107. Fondo de participaciones y otros fondos subordinados con vencimiento en la liquidación; 1070. Fondo de participaciones con vencimiento en la liquidación; 1071. Otros fondos subordinados con vencimiento en la liquidación". Por el contrario, cuando no se cumplan los mencionados requisitos y, por consiguiente, se califiquen las participaciones especiales como un pasivo financiero, se contabilizarán en la cuenta "1714. Acreedores por fondos capitalizados a largo plazo", cuando su vencimiento sea en el largo plazo, y en la cuenta "5214. Acreedores por fondos capitalizados a corto plazo", cuando su vencimiento sea en el corto plazo.

Una vez definidos los aspectos de índole jurídica y contable más relevantes de las obligaciones, participaciones especiales y títulos participativos, se procede a mostrar los resultados obtenidos conforme a la metodología planteada.

\section{Resultados obtenidos}

Conforme a la metodología planteada, la búsqueda de empresas en el SABI, de acuerdo con los criterios propuestos arroja un total de 486 entidades asociativas (cooperativas y SAT), 2.053 sociedades anónimas y 3.193 sociedades de responsabilidad limitada. De cada una de estas categorías se ha seleccionado el top 10, de acuerdo con su volumen de negocio, y se han extraído los datos referentes a la emisión de títulos objeto de estudio en los años 2015 a 2019 (tablas 3, 4 y 5). 
Tabla. 3. Sociedades cooperativas del sector agroalimentario con mayor volumen de facturación en el año 2019 en España según Base de Datos SABI

\begin{tabular}{|c|c|c|c|}
\hline Nombre & $\begin{array}{l}\text { Obligaciones y } \\
\text { otros valores } \\
\text { negociables a largo } \\
\text { plazo (2015-2019) } \\
\text { mil EUR }\end{array}$ & $\begin{array}{l}\text { Obligaciones y otros } \\
\text { valores negociables } \\
\text { a corto plazo (2015- } \\
\text { 2019) mil EUR }\end{array}$ & $\begin{array}{l}\text { Deudas con } \\
\text { características } \\
\text { especiales a largo } \\
\text { plazo (2015- } \\
\text { 2019) mil EUR }\end{array}$ \\
\hline DCOOP S.C. AND & n.p. & n.p. & $\begin{array}{ll}11.265 & (2019) ; \\
10.818 \quad(2018) ; \\
13.217 \quad(2017) ; \\
5.536 \text { (2016) }\end{array}$ \\
\hline ANECOOP S.C. & n.p. & n.p. & $\begin{array}{ll}949(2017) ; 850 \\
(2016) ; & 788 \\
(2015) & \end{array}$ \\
\hline A. N. S. C. & n.p. & n.p. & $\begin{array}{ll}3.618 & (2019) ; \\
2.831 & (2018) ; \\
3.287 & (2017) ; \\
3.069 & (2016) ;\end{array}$ \\
\hline $\begin{array}{l}\text { COOP } \\
\text { OURENSANAS S. } \\
\text { C. GALEGA }\end{array}$ & n.p. & n.p. & n.p. \\
\hline $\begin{array}{l}\text { COVIRAN } \\
\text { SOCIEDAD } \\
\text { COOPERATIVA } \\
\text { ANDALUZA } \\
\end{array}$ & n.p. & n.p. & n.p. \\
\hline $\begin{array}{l}\text { SCA GANADERA } \\
\text { DEL VALLE DE } \\
\text { LOS } \\
\text { PEDROCHES }\end{array}$ & n.p. & n.p. & $\begin{array}{l}44.690 \quad(2018) ; \\
44.969 \quad(2017) ; \\
39.835(2016) ; \\
37.621(2015)\end{array}$ \\
\hline $\begin{array}{lr}\text { UNICA } & \text { GROUP } \\
\text { S.C. ANDALUZA }\end{array}$ & n.p. & n.p. & n.p. \\
\hline $\begin{array}{l}\text { S.C. LTDA BAJO } \\
\text { DUERO }\end{array}$ & n.p. & n.p. & $\begin{array}{l}1.867(2018) ; \\
1.769(2017) ; \\
1.659(2016) ; \\
1.601(2015)\end{array}$ \\
\hline $\begin{array}{l}\text { FRIBIN S. A. T. } \\
\text { N. } 1269 \text { R. L. }\end{array}$ & n.p. & n.p. & n.p. \\
\hline $\begin{array}{l}\text { AGROPECUARIA } \\
\text { DE GUISSONA } \\
\text { S.C.L. } \\
\end{array}$ & n.p. & n.p. & $347(2015)$ \\
\hline
\end{tabular}

n.p. no presenta

Fuente: Elaboración propia a partir de la base de datos SABI.

Es pertinente destacar que el top 10 obtenido a partir de la Base de datos SABI difiere de los resultados recopilados en el Observatorio Socioeconómico del Cooperativismo Agroalimentario Español (OSCAE, 2019), ya que en el mencionado informe se recogen los volúmenes de facturación del año 2018 y la tabla 3 ha sido elaborada con los datos disponibles del año 2019. 
Tabla. 4. Sociedades anónimas del sector agroalimentario con mayor volumen de facturación en el año 2019 en España según Base de Datos SABI

\begin{tabular}{|c|c|c|c|}
\hline Nombre & $\begin{array}{l}\text { Obligaciones y otros } \\
\text { valores negociables a } \\
\text { largo plazo (2015- } \\
\text { 2019) mil EUR }\end{array}$ & $\begin{array}{l}\text { Obligaciones y } \\
\text { otros } \quad \text { valores } \\
\text { negociables a corto } \\
\text { plazo (2015-2019) } \\
\text { mil EUR }\end{array}$ & $\begin{array}{l}\text { Deudas con } \\
\text { características } \\
\text { especiales a largo } \\
\text { plazo (2015- } \\
\text { 2019) mil EUR }\end{array}$ \\
\hline NESTLE ESPAÑA SAU & n.p. & n.p. & n.p. \\
\hline $\begin{array}{lc}\text { COMPAÑIA } & \text { DE } \\
\text { DISTRIBUCION } & \\
\text { INTEGRAL LOGISTA SA }\end{array}$ & n.p. & n.p. & n.p. \\
\hline $\begin{array}{l}\text { CORPORACION } \\
\text { ALIMENTARIA } \\
\text { GUISSONA，SOCIEDAD } \\
\text { ANONIMA }\end{array}$ & n.p. & n.p. & n.p. \\
\hline $\begin{array}{l}\text { EL POZO } \\
\text { ALIMENTACION SA }\end{array}$ & n.p. & n.p. & n.p. \\
\hline $\begin{array}{l}\text { MAKRO } \\
\text { AUTOSERVICIO } \\
\text { MAYORISTA SAU }\end{array}$ & n.p. & n.p. & n.p. \\
\hline $\begin{array}{lr}\text { GENERAL } & \text { MARKETS } \\
\text { FOOD } & \text { IBERICA } \\
\text { SOCIEDAD } & \text { ANONIMA. }\end{array}$ & n.p. & n.p. & n.p. \\
\hline $\begin{array}{lr}\text { CAMPOFRIO } & \text { FOOD } \\
\text { GROUP, } & \text { SOCIEDAD } \\
\text { ANONIMA } & \end{array}$ & $\begin{array}{l}396.108(2017) \\
494.083(2016) \\
493.069(2015)\end{array}$ & n.p. & n.p. \\
\hline EBRO FOODS, SA & n.p. & n.p. & n.p. \\
\hline $\begin{array}{l}\text { CONDIS } \\
\text { SUPERMERCATS SA }\end{array}$ & n.p. & n.p. & n.p. \\
\hline $\begin{array}{l}\text { RIVASAM } \\
\text { INTERCONTINENTAL } \\
\text { SA }\end{array}$ & n.p. & n.p. & n.p. \\
\hline
\end{tabular}

n.p. no presenta

Fuente: Elaboración propia a partir de la base de datos SABI. 
Tabla. 5. Sociedades de responsabilidad limitada del sector agroalimentario con mayor volumen de facturación en el año 2019 en España según Base de Datos SABI.

\begin{tabular}{|c|c|c|c|}
\hline Nombre & $\begin{array}{l}\text { Obligaciones y otros } \\
\text { valores negociables } \\
\text { a largo plazo (2015- } \\
\text { 2019) mil EUR }\end{array}$ & $\begin{array}{l}\text { Obligaciones y } \\
\text { otros valores } \\
\text { negociables r a } \\
\text { corto plazo (2015- } \\
\text { 2019) mil EUR }\end{array}$ & $\begin{array}{l}\text { Deudas con } \\
\text { características } \\
\text { especiales a } \\
\text { largo plazo } \\
(2015-2019) \\
\text { mil EUR }\end{array}$ \\
\hline $\begin{array}{l}\text { FLORETTE } \\
\text { FconsulOOD SERVICE } \\
\text { IBERICA SL. }\end{array}$ & n.p. & n.p. & n.p. \\
\hline $\begin{array}{l}\text { COCA-COLA } \\
\text { EUROPEAN } \\
\text { PARTNERS IBERIA } \\
\text { SL. } \\
\end{array}$ & n.p. & n.p. & n.p. \\
\hline CARGILL SLU & n.p. & n.p. & n.p. \\
\hline GADISA RETAIL SL & n.p. & n.p. & n.p. \\
\hline $\begin{array}{l}\text { COMPAÑIA } \\
\text { NORTEÑA DE } \\
\text { BEBIDAS GASEOSAS } \\
\text { NORBEGA SOCIEDAD } \\
\text { LIMITADA }\end{array}$ & n.p. & n.p. & n.p. \\
\hline $\begin{array}{l}\text { COBEGA } \\
\text { EMBOTELLADOR } \\
\text { SLU }\end{array}$ & n.p. & n.p. & n.p. \\
\hline $\begin{array}{l}\text { PESCANOVA ESPAÑA } \\
\text { SL. }\end{array}$ & n.p. & n.p. & n.p. \\
\hline $\begin{array}{ll}\text { PHILIP } & \text { MORRIS } \\
\text { SPAIN SL } & \end{array}$ & n.p. & n.p. & n.p. \\
\hline $\begin{array}{l}\text { MONDELEZ ESPAÑA } \\
\text { COMMERCIAL SL. }\end{array}$ & n.p. & n.p. & n.p. \\
\hline MERCARTABRIA SL & n.p. & n.p. & n.p. \\
\hline
\end{tabular}

n.p. no presenta

Fuente: Elaboración propia a partir de la base de datos SABI.

Recopilada la información, conforme a la metodología planteada, se observan notables diferencias entre los tres tipos de sociedades analizadas. En primer lugar, llama la atención la cifra de facturación de las sociedades de responsabilidad limitada ya que superan con creces a las sociedades anónimas y a las cooperativas o SAT. La mayor de las sociedades de responsabilidad limitada, por nivel de facturación según los datos recopilados del año 2019, supera los 9.000 .000 mil EUR, cifra que deja muy por detrás a la mayor de las sociedades anónimas que factura algo más de 2.000 .000 mil EUR y también supera a la mayor de las sociedades cooperativas que ronda los 1.000 .000 mil EUR. Sin embargo, esta situación no es equivalente si se analiza la cuantía total de activos de las sociedades ya que, en este caso, las sociedades anónimas superan al resto, por cuanto que la mayor de las sociedades anónimas supera los 4.500 .000 mil EUR de activos, quedándose la mayor de las sociedades de responsabilidad limitada en algo más de 3.600.000 mil EUR y la mayor de las cooperativas en poco más de 700.00 mil EUR. Las diferencias de volumen de facturación y total de activos se mantienen en los primeros niveles y se igualan en los niveles intermedios. Estos datos ponen de manifiesto que el volumen facturación y tamaño de las sociedades de capital del sector agroalimentario supera notablemente al volumen de facturación y tamaño de las sociedades cooperativas.

A colación con lo anterior, interesa analizar el nivel de endeudamiento de las sociedades analizadas, para tratar de evidenciar si la utilización de financiación ajena es mayor que la aportada por los socios (aportaciones sociales) o la generada por la propia cooperativa (excedentes, fondos o reservas). Llama la atención observar que las sociedades cooperativas ostentan unos niveles de endeudamiento que, en bastantes de los casos seleccionados, superan el 75\% llegando, en ocasiones, a superar el 90\%, lo que constata que 
lejos de bastarles la financiación de sus socios y los excedentes generados, recurren en gran medida a la financiación ajena.

Resultan llamativos estos elevados niveles de endeudamiento de las sociedades cooperativas, que deberían ser más característicos de las sociedades de capital, que tradicionalmente se han caracterizado por una más elevada asunción de riesgo por parte de los socios. Ahora bien, sentado lo que acontece se procede a analizar si parte de esa financiación se corresponde con la otorgada por las entidades de crédito, proveedores y acreedores (incluidos los saldos que se refieren a los propios socios por entrega de sus cosechas) o si, por el contrario, además de esta financiación, existen obligaciones, participaciones especiales o títulos participativos en los balances de las sociedades seleccionadas.

Para ello se dispone de la información relativa a las cuentas de obligaciones y otros valores negociables a largo y corto plazo y deuda con características especiales a largo plazo. De la información obtenida se observa que la mayoría de las cooperativas ostenta saldo en el apartado de Deuda con características especiales a largo plazo. Esta cuenta, conforme a la ya mencionada Orden EHA/3360/2010, recoge el saldo de los socios que representando el $1 \%$ del capital social han causado baja como socios, y tienen pendiente de reembolso u aportación, debiéndose, en consecuencia, considerarse como un pasivo financiero. De manera que el saldo de esta cuenta no representará la emisión de las obligaciones, participaciones especiales o títulos participativos. Por tanto, de haberse realizado una emisión de obligaciones, títulos participativos o participaciones especiales, debería haberse contemplado en el apartado de obligaciones y otros valores negociables a largo o plazo. Como se observa en la Tabla 3, ninguna de las sociedades cooperativas presentadas reúne saldo en esa cuenta. Sin embargo, sí que existe una sociedad cooperativa incluida en el top 50 de España, por volumen de facturación según los datos de SABI 2019, llamada COOP PROVINCIAL AGRARIA Y GANADERA SAN ISIDRO S.C. ANDALUZA, que ostentaría un saldo en esta cuenta de 16.706 mil EUR en el año 2018, siendo ésta la única en este colectivo. La lectura general que se puede extraer es que si las grandes sociedades cooperativas, a excepción de COOP PROVINCIAL AGRARIA Y GANADERA SAN ISIDRO S.C. ANDALUZA, con grandes expectativas de crecimiento y gran volumen de negocio no hacen uso de las obligaciones, participaciones especiales y títulos participativos, el resto de sociedades cooperativas más modestas, por volumen de actividad y tamaño, tampoco las utilizarán, por lo que, atenderán a sus obligaciones de pago mediante las aportaciones de sus socios y préstamos bancarios.

En cuanto a las sociedades de responsabilidad limitada y anónimas analizadas, sorprende observar que tampoco han emitido títulos como método alternativo de financiación, con la excepción de una sociedad anónima denominada CAMPOFRIO FOOD GROUP, SOCIEDAD ANONIMA que, en los años 2017, 2016 y 2015 presentaba un saldo de 396.108 mil EUR, 494.083 mil EUR y 493.069 mil EUR respectivamente en la cuenta de obligaciones y otros valores negociables a largo plazo.(Tabla 4) Por todo ello, se puede afirmar, que, en general -salvo las dos excepciones comentadas, las sociedades cooperativas, las anónimas y las de responsabilidad limitada analizadas del sector agroalimentario español, no utilizan las obligaciones, participaciones especiales y títulos participativos como método alternativo a la denominada financiación tradicional.

\section{Conclusiones}

El presente trabajo trataba de verificar si las sociedades cooperativas utilizan las obligaciones, participaciones especiales y títulos participativos como método alternativo de financiación. Para ello, en primer lugar, se ha realizado un breve análisis jurídico sobre este tipo de instrumentos financieros, habiéndose observado la dispersión jurídica existente en materia de cooperativas, en la que una misma cuestión puede estar regulada de 16 maneras diferentes. Como ya se ha señalado en múltiples ocasiones por parte de la academia, sería interesante promover una mayor armonización en el ámbito cooperativo que permitiera a las Comunidades Autónomas desarrollar determinados aspectos concretos, evitando discrepancias a la hora de interpretar una misma cuestión.

Una vez expuestos los elementos diferenciadores de cada uno de los instrumentos financieros objeto de estudio en el presente trabajo, se ha podido observar que la práctica totalidad de sociedades analizadas cooperativas, sociedades de responsabilidad limitada y sociedades anónimas- no utilizan este tipo de instrumentos financieros. Ello se infiere del estudio de sus partidas contables extraídas de la Base de datos SABI de donde podemos extraer que la forma de financiación utilizada por las sociedades cooperativas se centra eminentemente en los sistemas tradicionales de financiación, tales como las aportaciones de sociales y la financiación otorgada por las entidades de crédito.

Esto nos llama especialmente la atención toda vez que el propósito del legislador, cuando introdujo estos instrumentos financieros, era dotar de más fuentes de financiación a las sociedades cooperativas y así poder tener un amplio abanico de posibilidades para poder captar recursos financieros de terceros (Pastor Sempere, 2002). Con los datos obtenidos en el presente trabajo se concluye que el propósito del legislador no se ha cumplido. Y ello nos hace reflexionar acerca de si estos instrumentos financieros están regulados de forma 
que otorguen suficiente libertad a la sociedad cooperativa que los emite, en el sentido de poder configurarlos acorde a sus necesidades de financiación.

A pesar de la flexibilidad normativa, las principales fuentes de financiación de las sociedades cooperativas siguen siendo las tradicionales y, además, se observa una gran dependencia de la financiación otorgada por las entidades de crédito que en la mayoría de los casos supera las aportaciones de los socios. Las causas que podrían motivar su no utilización quizás pueden deberse a un desconocimiento de su régimen jurídico, junto con las implicaciones que su emisión podría tener en el gobierno de la cooperativa; sin embargo, carecemos de información para afirmarlo.

Esta situación se ha intentado contrastar con las sociedades anónimas y de responsabilidad limitada que operan en el sector agroalimentario. Los datos obtenidos son muy similares, puesto que las sociedades de capital del sector tampoco hacen uso de este tipo de instrumentos financieros. Ello nos permite pensar que, quizás, la utilización de este tipo de instrumentos financieros no dependa de la tipología societaria sino del sector de actividad al que pertenecen las entidades. Pese a ello, se ha comprobado que en el sector agroalimentario los niveles de endeudamiento de las sociedades son muy elevados en todos los tipos de sociedades analizados. La emisión de títulos, no solo en las cooperativas sino también en las sociedades de capital, permitiría diversificar los niveles de riesgo en diferentes agentes y titulares, evitando que todo el peso del endeudamiento recaiga sobre los socios.

A modo de reflexión final, y aun reconociendo el posible sesgo introducido por la limitación de los datos utilizados conforme a la metodología de trabajo planteada, las conclusiones y resultados reflejados en el presente trabajo testimonian de forma clara un uso muy reducido o nulo de estos instrumentos financieros. La reducción del sesgo, y la indagación de las causas por las que las cooperativas no hacen el uso que cabría esperar de dichos instrumentos, aconseja utilizar otros métodos de recopilación de datos de carácter cualitativo, como el método Delphi, mediante la realización de encuestas que permitieran acceder a mayores fuentes de información de datos sobre las sociedades cooperativas.

En otro orden de cosas, otro elemento que conviene considerar es que las obligaciones, participaciones especiales y títulos participativos son títulos que pueden considerarse ya antiguos, ya que datan de mediados de la década de los 90 (Pastor Sempere, 2002). Resultaría por tanto recomendable, dado el elevado nivel de endeudamiento de estas entidades, revisar la legislación aplicable en materia de cooperativas para abrirlas a la emisión de nuevos instrumentos financieros, desde el respeto a los principios y valores cooperativos, y en especial al principio democrático, que asegura el mantenimiento del poder de decisión en la base social, sin interferencias de terceros.

\section{Referencias bibliográficas}

Battilani, P. and Schröter, H. G. (2012) Demutualization and its problems, en Battilani, P. and Schröter, H. G., The Cooperative Business Movement, 1950 to the Present, Cambridge University Press, pp. 150-172.

Bekkum, O. F. Van and Bijman, J. (2006) Innovations in cooperative ownership: Converted and hybrid listed cooperatives, 7th International Conference on Management in AgriFood Chains and Networks.

Bernard T., Taffesse A. S., Gabre-Madhin E. (2008) Impact ofcooperatives on smallholders' commercialization behavior: evidence from Ethiopia, Agricultural Economics, $\mathrm{N}^{\circ} 39$, pp. 147-161.

Chaddad, F. R. and Cook, M. L. (2004) Understanding new cooperative models: An ownership-control rights typology, Review of Agricultural Economics, 26(3), pp. 348-360.

Cook, M. (1995) The future of US agricultural cooperatives A neo-institutional approach, American Journal of Agricultural Economics, $\mathrm{N}^{\circ} 77$, pp. 1153-1159.

Cook, M. L. and Iliopoulos, C. (2000) Ill-defined property rights in collective action: the case of US agricultural cooperatives, en Ménard, C. (ed.) Institutions, Contracts and Organizations. Edward Elgar, pp. 335-348.

Fajardo García, I. (2005) La masa activa y pasiva en el concurso de la cooperativa, en AA.VV., Estudios sobre la Ley Concursal. Libro Homenaje a Manuel Olivencia, Tomo V, Madrid, pp. 5235-5280.

Fajardo García G. (2011) Cooperativas: Régimen jurídico y Fiscal, Tirant lo Blanch.

Gadea, E., Sacristán, F., Vargas Vasserot, C. (2009) Régimen jurídico de la sociedad cooperativa del siglo XXI. Realidad actual y propuesta de reforma, Madrid, Dykinson.

Gadea, E. (2003) La financiación de las Sociedades Cooperativas: un estudio desde la perspectiva de la Ley de cooperativas del País Vasco, Boletín Internacional de Derecho Cooperativo, № 37, pp. 37-54.

García de Enterría, J. (2006) La Sociedad Anónima: las obligaciones, en AA.VV., Curso de Derecho Mercantil, (UríaMenéndez), T.I, $2^{\mathrm{a}}$ edición, pp.1063-1086.

Grashuis, J. (2018) An exploratory study of cooperative survival: Strategic adaptation to external developments, Sustainability (Switzerland), 10(3).

Harte, L. (1997) Creeping Privatisation of Irish Co-operatives: A Transaction Cost Explanation, Strategies and Structures in The Agro-Food Industries, pp. 31-53.

Ito J., Bao Z., Su Q. (2012) Distributional effects of agricultural cooperatives in China: Exclusion of smallholders and potential gains on participation, Food Policy, No 37, pp. 700-709. 
Macías Ruano, A. J. (2016) Las sociedades cooperativas y la adaptación de sus principios al mercado, Editorial Cajamar Caja Rural.

Martín Castro, M. ${ }^{a}$ Paz (2019) Capítulo VII. Régimen económico, en Peinado Gracia, J.I., Vázquez Ruano, T., Tratado de derecho de sociedades cooperativas. Tomo I, $2^{\mathrm{a}}$ edición, Tirant lo Blanch.

Meliá Martí, E., Carnicer Andrés, P., Juliá Igual, J. F. (2018) El gobierno de las sociedades cooperativas agroalimentarias españolas. Modelos de referencia y casos empresariales. Ed. Monografías Cajamar Caja Rural.

Macías Ruano, A. J. (2016) Las sociedades cooperativas y la adaptación de sus principios al mercado, Editorial Cajamar, Caja Rural.

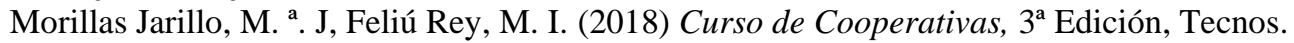

Nilsson, J. (1999) Co-operative Organisational Models as Reflections of the Business Environments; The Finnish Journal of Business Economics, 4 (Special Issue: The Role of Cooperative Entrepreneurship in the Modern Market Environment), pp. 449-470.

Nilsson, Jerker; Svendsen, G.L.H; Svendsen, G. (2012) Are Large and Complex Agricultural Cooperatives Losing Their Social Capital?, Agribusiness, 28(2), pp. 187-204.

Ortmann, G. F. and King, R. P. (2007) Agricultural cooperatives I: History, theory and problems, Agrekon, 46(1), pp. $18-46$.

Observatorio Socioeconómico del Cooperativismo Agroalimentario Español (OSCAE) (2019) El cooperativismo agroalimentario español. Informe socioeconómico 2018, Ministerio de Agricultura, Pesca y Alimentación, Cooperativas agroalimentarias de España.

Pastor Sempere, C. (2002) Los recursos propios en las sociedades cooperativas. Edersa, Madrid.

Pastor Sempere, C. (2011) Régimen Económico y Financiero I. Capital social, reservas y financiación, en Fajardo García, G., Cooperativas: Régimen Jurídico y Fiscal, Tirant lo Blanch.

Peinado Gracia, J.I., Vázquez Ruano, T. (2019) Tratado de derecho de sociedades cooperativas. Tomo I, $2^{\mathrm{a}}$ edición, Tirant lo Blanch, Tomos I y II.

Pendón Meléndez, M.A. (2019) Capítulo VII. Régimen económico, en Peinado Gracia, J. I., Vázquez Ruano, T., Tratado de derecho de sociedades cooperativas. Tomo I, $2^{\text {a }}$ edición, Tirant lo Blanch, pp. 682-751.

Rocafort Nicolau, A. (2010) La financiación cooperativa mediante la emisión de títulos participativos. CIRIEC-España, Revista de Economía Pública, Social y Cooperativa, N. ${ }^{\circ}$ 68, pp. 141-166.

Royer, J. S. (1990) Cooperative Organizational Strategies: A Neo-Institutional Digest, Journal of Cooperatives.

Vicent Chuliá, F. (2012) Introducción al Derecho Mercantil, 23 a edición, Volumen I, Tirant lo Blanch, Valencia. 